\title{
Improving the Spatial Alignment in PET/CT Using Amplitude-Based Respiration-Gated PET and Respiration-Triggered CT
}

\author{
Charlotte S. van der $\operatorname{Vos}^{1,2}$, Willem Grootjans ${ }^{1}$, Dustin R. Osborne ${ }^{3}$, Antoi P.W. Meeuwis ${ }^{1}$, James J. Hamill ${ }^{4}$, \\ Shelley Acuff ${ }^{3}$, Lioe-Fee de Geus-Oei ${ }^{1,2,5}$, and Eric P. Visser ${ }^{1}$ \\ ${ }^{I}$ Department of Radiology and Nuclear Medicine, Radboud university medical center, Nijmegen, The Netherlands; ${ }^{2}$ MIRA Institute for \\ Biomedical Technology and Technical Medicine, University of Twente, Enschede, The Netherlands; ${ }^{3}$ Department of Radiology, \\ University of Tennessee Graduate School of Medicine, Knoxville, Tennessee; ${ }^{4}$ Siemens Healthcare, Knoxville, Tennessee; and \\ ${ }^{5}$ Department of Radiology, Leiden University Medical Center, Leiden, The Netherlands
}

Respiratory motion during PET can cause inaccuracies in the quantification of radiotracer uptake, which negatively affects PETguided radiotherapy planning. Quantitative accuracy can be improved by respiratory gating. However, additional miscalculation of standardized uptake value (SUV) in PET images can be caused by inappropriate attenuation correction due to a spatial mismatch between gated PET and CT. In this study, the effect of respirationtriggered CT on the spatial match between CT and amplitude-based respiration-gated PET images is investigated. Methods: ${ }^{18} \mathrm{~F}-\mathrm{FDG}$ $\mathrm{PET} / \mathrm{CT}$ was performed in 38 patients. Images were acquired on 2 PET/CT scanners, one without and one with continuous bed motion during PET acquisition. The amplitude limits of the amplitude-based respiration-gated PET were used for the respiration-triggered sequential low-dose CT. Both standard (spiral) and triggered CT scans were used to reconstruct the PET data. Spatial mismatch was quantified using the position difference between the lung-liver boundary in PET and CT images, the distance between PET and CT lung lesions' centroids, and the amount of overlap of lesions indicated by the Jaccard similarity coefficient. Furthermore, the effect of attenuation correction was quantified by measuring SUVs in lung lesions. Results: For triggered CT, the average distance between the lung-liver boundary in PET and CT was significantly reduced $(4.5 \pm 6.7 \mathrm{~mm})$ when compared with standard CT $(9.2 \pm 8.1 \mathrm{~mm})$ $(P<0.001)$. The mean distance between the lesions' centroids in PET and CT images was $6.3 \pm 4.0$ and $5.6 \pm 4.2 \mathrm{~mm}(P=0.424)$, for the standard and triggered $C T$, respectively. Similarly, the Jaccard similarity coefficient was $0.30 \pm 0.21$ and $0.32 \pm 0.20(P=0.609)$ for standard and triggered CT, respectively. For 6 lesions, there was no overlap of PET and CT when the standard CT was used; compared with the triggered CT, these lesions showed (partial) overlap. The maximum and mean SUV increase of the PET/CT compared with the PET/triggered CT was $5.7 \% \pm 11.2 \%(P<0.001)$ and $6.1 \% \pm$ $10.2 \%(P=0.001)$, respectively. Conclusion: Amplitude-based respiration-gated PET in combination with respiration-triggered CT resulted in a significantly improved match in the area of the liver dome and a significantly higher SUV for lung lesions. However, lesions in the lungs did not show a consistent improvement in spatial match.

Received Jul. 2, 2015; revision accepted Sep. 3, 2015.

For correspondence or reprints contact: Charlotte S. van der Vos, Department of Radiology and Nuclear Medicine, Radboud university medical center, P.O. Box 9101, 6500 HB Nijmegen, The Netherlands.

E-mail: charlotte.vandervos@radboudumc.nl

Published online Sep. 17, 2015.

COPYRIGHT (c) 2015 by the Society of Nuclear Medicine and Molecular Imaging, Inc.
Key Words: amplitude-based optimal respiratory gating; lung tumors; image quantification in PET; respiration-triggered CT; spatial alignment

J Nucl Med 2015; 56:1817-1822

DOI: $10.2967 /$ jnumed.115.163055

$\mathbf{T}$ he combination of PET and CT is widely used in oncology (1-3). PET/CT imaging provides anatomic and metabolic information, which is important for detection, localization, and characterization of the disease $(2,3)$. Furthermore, quantitative indices in PET, such as standardized uptake value (SUV) $(4,5)$, metabolic volume $(6,7)$, and total lesion glycolysis $(5-7)$, can be used to provide prognostic information and predict and monitor therapy response (4-7).

Because of the relatively long acquisition time of PET, patients are usually instructed to breathe freely during the PET acquisition, resulting in respiratory motion artifacts $(3,8,9)$. Within the thorax and upper abdomen, significant blurring can occur, resulting in an overestimation of the size of structures and an underestimation of SUV $(3,8-10)$. If the PET image is not corrected for respiratory motion, the artifacts can introduce diagnostic uncertainties and inaccuracies in quantitative indices and delineation of target volumes, which can result in an unnecessarily high radiation dose to normal tissue in radiotherapy planning as well as inadequate monitoring of therapy response $(3,10,11)$. Methods are available to reduce these respiratory motion artifacts in PET images, commonly referred to as respiratory gating. These methods typically rely on the use of PET data acquired at specific time intervals, resulting in a motion-reduced image reconstruction (11). Because the low-dose (LD) CT is usually performed without breathing instructions, a significant spatial mismatch between PET and CT can occur for structures that move during respiration $(11,12)$. The LD CT is used for attenuation correction of the PET image, which is one of the most important correction stages in PET (9). A mismatch between PET and CT can lead to quantitative inaccuracies in PET (11). In sum, spatial matching of PET and CT is important, especially for lesions located near the transition of anatomic regions with different densities (e.g., the lower lung and liver dome) $(13,14)$. 
Several methods have been proposed to improve the spatial matching of LD CT with PET, for example, breathing instructions $(12,15)$ or rigid/nonrigid transformations (16). Van Elmpt et al. suggested that respiration-triggered CT could be used for this purpose (17). In the present study, we pursue the triggered CT concept, comparing results based on spiral and triggered CT for improving spatial matching of CT with amplitude-based optimal respiration-gated PET imaging.

\section{MATERIALS AND METHODS}

\section{Patients}

Whole-body ${ }^{18} \mathrm{~F}-\mathrm{FDG}$ PET/CT imaging was performed in 38 patients with suspected lung cancer. Thirty patients were recruited at the Radboud university medical center (Radboudumc) and 8 patients at the University of Tennessee Medical Center (UT). The local Institutional Review Board of the Radboudumc waived the need to assess the protocol; nevertheless, all the patients signed informed consent. Data from the UT Medical Center were collected under a UT Institutional Review Board-approved protocol. Patient characteristics are summarized in Table 1.

\section{Patient Preparation}

At the Radboudumc, administration of ${ }^{18} \mathrm{~F}-\mathrm{FDG}$ was dependent on patient weight $\left(3.1 \pm 0.2 \mathrm{MBq} \cdot \mathrm{kg}^{-1}\right)$. The mean incubation time was $74 \mathrm{~min}$. At the UT, the administered amount of ${ }^{18} \mathrm{~F}-\mathrm{FDG}$ was independent of patient weight. The mean administered activity at this institute was $371 \pm 25 \mathrm{MBq}$, with a mean incubation time of $80 \mathrm{~min}$.

\section{PET Acquisition and Respiratory Gating}

The Biograph $40 \mathrm{mCT}$ PET/CT scanner (Siemens Healthcare) with extended field of view (TrueV) was used at the Radboudumc. This scanner is EANM Research Ltd. accredited (18). The Biograph 64 mCT Flow PET/CT scanner (Siemens Healthcare) was used at UT. PET images from both scanners were acquired using an optimized, amplitude-based respiratory gating algorithm (HD-Chest) integrated in the PET/CT software $(11,17)$. For the Biograph $40 \mathrm{mCT}$, the respiratory gating was performed on bed positions covering the thorax and upper abdomen. Gated and nongated bed positions were acquired during free breathing for 6 and $2 \mathrm{~min}$, respectively. The difference in acquisition time led to similar count densities after gating. For the Biograph 64 mCT Flow scanner (19), continuous-bed-motion scans were acquired using 3 ranges with 2 different table speeds. Through all areas other than the thorax and upper abdomen, a table speed of $1.5 \mathrm{~mm} / \mathrm{s}$ was used. When gating was applied, the table speed was slowed to $0.4 \mathrm{~mm} / \mathrm{s}$ to acquire count densities similar to those found in the standard $1.5 \mathrm{~mm} / \mathrm{s}$ ranges. On both scanners, a respiratory amplitude signal was acquired with an AZ-733V respiratory gating system (Anzai Medical Co, Ltd.) with a pressure sensor integrated in an elastic belt placed around the patient's abdomen. Respiratory gating was performed with a duty cycle of $35 \%$, providing a good balance between image quality and motion rejection (11). This was done with standard components in the PET/CT scanner, with digitized respiratory amplitude signals from AZ-733V electronics.

\section{PET Image Reconstruction}

The PET images for both scanners were reconstructed using a 3-dimensional ordered-subset expectation maximization algorithm with a spatially varying point-spread function incorporating time-offlight information (UltraHD PET). Image reconstruction was performed with 3 iterations and 21 subsets. The slice thickness of the PET reconstructions was matched with the attenuation-correction CT. Postreconstruction filtering was performed using a 3-dimensional Gaussian filter kernel with a full width at half maximum of $3.0 \mathrm{~mm}$. A transaxial matrix size of $400 \times 400$ was used for the PET reconstructions of the Biograph 40 mCT scanner; for the Biograph 64 mCT Flow scanner a matrix size of $200 \times 200$ was used.

\section{Standard Free-Breathing LD Spiral CT Imaging}

A standard LD spiral CT (standard CT) was acquired for attenuation correction and anatomic reference. This standard CT was acquired with a free-breathing protocol. For the Biograph $40 \mathrm{mCT}$ scanner, the $\mathrm{x}$-ray tube voltage was chosen using CARE $\mathrm{kV}$, with a reference tube voltage of $100 \mathrm{kV}$. For the Biograph $64 \mathrm{mCT}$ Flow, $120 \mathrm{kV}$ was used in each case. The tube current for both scanners was modulated using CARE Dose4D (Siemens), with a reference tube current of $50 \mathrm{mAs}$. CT images were made with a $0.5 \mathrm{~s}$ tube rotation time and $16 \times 1.2 \mathrm{~mm}$ collimation; a reconstruction with an increment

TABLE 1

Summary of Patient Characteristics

\begin{tabular}{|c|c|c|}
\hline Characteristic & $\begin{array}{l}\text { Patients scanned with step-and-shoot } \\
\text { method (Biograph } 40 \mathrm{mCT} \\
\text { scanner) (value } \pm \mathrm{SD} \text { ) }\end{array}$ & $\begin{array}{l}\text { Patients scanned with continuous-bed- } \\
\text { motion method (Biograph } 64 \mathrm{mCT} \\
\text { Flow scanner) (value } \pm \text { SD) }\end{array}$ \\
\hline \multicolumn{3}{|l|}{ Sex } \\
\hline Males & 19 & 5 \\
\hline Females & 11 & 3 \\
\hline Age (y) & $68.7 \pm 8.6$ & $66.0 \pm 6.2$ \\
\hline Weight (kg) & $81.5 \pm 17.3$ & $68.6 \pm 18.7$ \\
\hline \multicolumn{3}{|l|}{ Location of lesion } \\
\hline Upper lobes & 19 & 4 \\
\hline Middle and lower lobes & 13 & 9 \\
\hline Hilum & 8 & 3 \\
\hline Duration of triggered CT (min) & $3.1 \pm 1.6$ & $1.7 \pm 0.7$ \\
\hline Length of triggered CT (cm) & $46.8 \pm 7.0$ & $32.0 \pm 3.9$ \\
\hline
\end{tabular}

Lesions showing invasive growth into or attachment to large structures (arteries, veins, and main bronchi) of lung hilum were assigned to hilum group. 


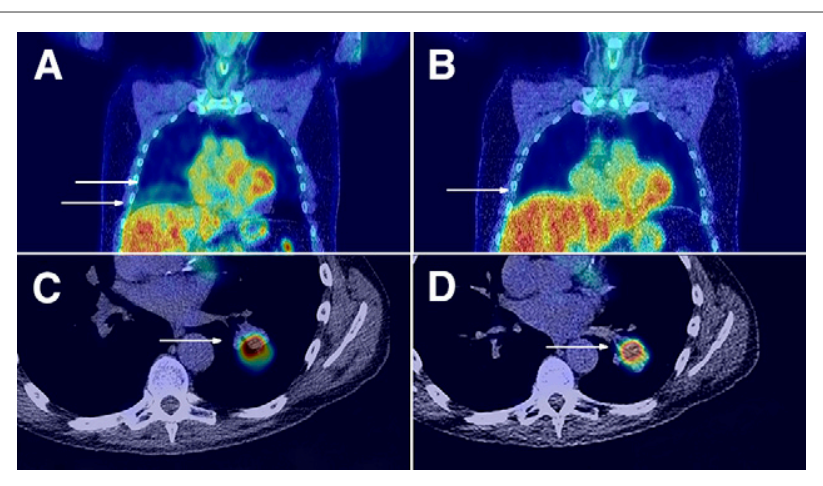

FIGURE 1. Two patients with non-small cell lung cancer, 1 with squamous cell carcinoma ( $A$ and $B$ ) and 1 with adenocarcinoma ( $C$ and $D)$. First column ( $A$ and $C$ ) depicts coronal $(A)$ and axial $(C)$ plane of standard CT fused with the respiration-gated PET image, reconstructed with $35 \%$ duty cycle. Second column (B and D) depicts same coronal (B) and axial (D) plane of triggered CT fused with corresponding gated PET images, reconstructed with $35 \%$ duty cycle. Both patients were scanned on Biograph $40 \mathrm{mCT}$ scanner. Both patients show improvement in match between PET and CT when PET and triggered CT is compared with $\mathrm{PET}$ and standard $\mathrm{CT}$. PET and triggered $\mathrm{CT}(\mathrm{B}$ and $\mathrm{D})$ show perfect match as indicated by single arrows, whereas there is mismatch for PET and standard CT group (A and C). For coronal PET and standard CT scan (A), 2 arrows were used to indicate lung-liver boundary on PET and CT.

of $3 \mathrm{~mm}$ and a reconstructed slice width of $5.0 \mathrm{~mm}$ was made. In the case of the Biograph $40 \mathrm{mCT}$, additional reconstructions of $3.0 \mathrm{~mm}$ slice width were used to quantify the lung-liver boundary mismatch.

\section{Respiration-Triggered LD Sequential CT Imaging}

Patients received an additional respiration-triggered LD CT scan (triggered CT) covering the thorax, in which we prospectively used the defined amplitude range from the gating algorithm of the PET reconstructions. The respiration-triggered $\mathrm{CT}$ approach was not entirely integrated into the PET/CT scanner. Instead, AZ-733V electronics digitized the respiratory amplitudes and issued a trigger to the CT gantry, with the amplitude matched to the amplitudes determined by the gating algorithm (20). The CT scanner acquired a CT image in response to each trigger, then advanced to the next bed position (sequential mode). The time required to cover the thorax was between $1 \mathrm{~min}$ and several minutes, depending on the scan range and the respiration of the patient. For the Biograph $40 \mathrm{mCT}$ scanner, the tube current was chosen using CARE kV (Siemens), with a reference tube voltage of $100 \mathrm{kV}$. For the Biograph $64 \mathrm{mCT}$ Flow, $120 \mathrm{kV}$ was used for all patients. The tube current was modulated for both scanners using CARE Dose4D, with a reference tube current of $35 \mathrm{mAs}$ for the Biograph $40 \mathrm{mCT}$ scanner and $50 \mathrm{mAs}$ for the Biograph $64 \mathrm{mCT}$ Flow. An x-ray dose of the triggered CT scan was similar to a spiral CT with the same voltage and current settings.

At the Radboudumc, 2 acquisition settings were used for the triggered CT. For the first 6 patients, triggered CT scans were acquired using $5.0 \mathrm{~mm}$ slices $(1 \times 5.0 \mathrm{~mm})$, a $5 \mathrm{~mm}$ table feed, and a rotation time of $0.36 \mathrm{~s}$. Because the acquisition time was long for those patients and the slice thickness was large, we scanned the other patients with a larger table feed and a possibility to reconstruct the data with thinner slices. These patients were scanned with $4.8 \mathrm{~mm}$ slices $(16 \times 1.2 \mathrm{~mm})$, a $19 \mathrm{~mm}$ table feed, and a rotation time of $0.36 \mathrm{~s}$ for the Biograph $40 \mathrm{mCT}$ scanner. The $4.8 \mathrm{~mm}$ reconstructions were used for the attenuation correction and lesion delineation. Additional reconstructions were made with a slice width of $2.4 \mathrm{~mm}$ for the determination of lung-liver boundaries. At UT, a method similar to the second approach was used but with a slice thickness of $2.0 \mathrm{~mm}$.

\section{Image Analysis}

Analysis of the PET and the CT images was performed using the Inveon Research Workplace 4.1 software (Preclinical Solutions, Siemens Medical Solutions). Spatial alignment between PET and CT structures was quantified using 4 methods. The first method was quantification of the mismatch for the liver dome in the craniocaudal direction. The liver dome was determined visually in the axial plane. The most cranial plane in which the liver was visible was measured for the PET and the CT scan, and the difference in craniocaudal direction was reported. For CT, the lung setting (window center: -450 Hounsfield units; width: 1,500 Hounsfield units) was used. For the second method, the lung lesions were delineated in the PET and CT image to determine the alignment of these lesions. On the PET image, the lesions were delineated using a fixed-threshold region-growing segmentation algorithm. The threshold was set to $40 \%$ of the maximum SUV ( $\left.\mathrm{SUV}_{\text {max }}\right)$, which is recognized as a suitable threshold level for delineation of lung lesions $(11-13,21)$. The lesions in the CT images were manually delineated using the lung setting. These delineations were used to calculate the distance between the centroids of the lesions on PET and CT $(13,15)$. The third method to quantify the alignment was the Jaccard similarity coefficient $(9,12)$, defined as

$$
\mathrm{J}\left(\mathrm{VOI}_{\mathrm{CT}}, \mathrm{VOI}_{\mathrm{PET}}\right)=\frac{\mathrm{VOI}_{\mathrm{CT}} \cap \mathrm{VOI}_{\mathrm{PET}}}{\mathrm{VOI}_{\mathrm{CT}} \cup \mathrm{VOI}_{\mathrm{PET}}} .
$$

In this equation, VOI denotes a volume of interest. For the fourth method, the $\mathrm{SUV}_{\text {max }}$ and mean SUV ( $\mathrm{SUV}_{\text {mean }}$ ) were compared on both PET reconstructions. Lesions were excluded if the segmentation was not possible on either PET or CT.

TABLE 2

Results of Analyses of Spatial Alignment for Both Patient Groups

\begin{tabular}{lccc}
\hline \multicolumn{1}{c}{ Parameter } & Standard CT and PET & Triggered CT and PET & $P$ \\
\hline Mismatch of lung-liver boundary $(\mathrm{mm})$ & $9.2 \pm 8.1$ & $4.5 \pm 6.7$ & $<0.001^{*}$ \\
Average distance between lesion centroids $(\mathrm{mm})$ & $6.3 \pm 4.0$ & $5.6 \pm 4.2$ & 0.424 \\
Jaccard similarity coefficient & $0.30 \pm 0.21$ & $0.32 \pm 0.20$ & 0.609 \\
SUV $_{\max }\left(\mathrm{g} / \mathrm{cm}^{3}\right)$ & $10.5 \pm 6.7$ & $10.9 \pm 6.7$ & $<0.001^{*}$ \\
$\operatorname{SUV}_{\text {mean }}\left(\mathrm{g} / \mathrm{cm}^{3}\right)$ & $6.1 \pm 4.0$ & $6.4 \pm 4.0$ & $0.001^{*}$
\end{tabular}

*Significant difference between 2 groups.

Numbers are averages and SD for all patients (i.e., scanned on Biograph 40 mCT and on Biograph 64 mCT Flow scanner). 


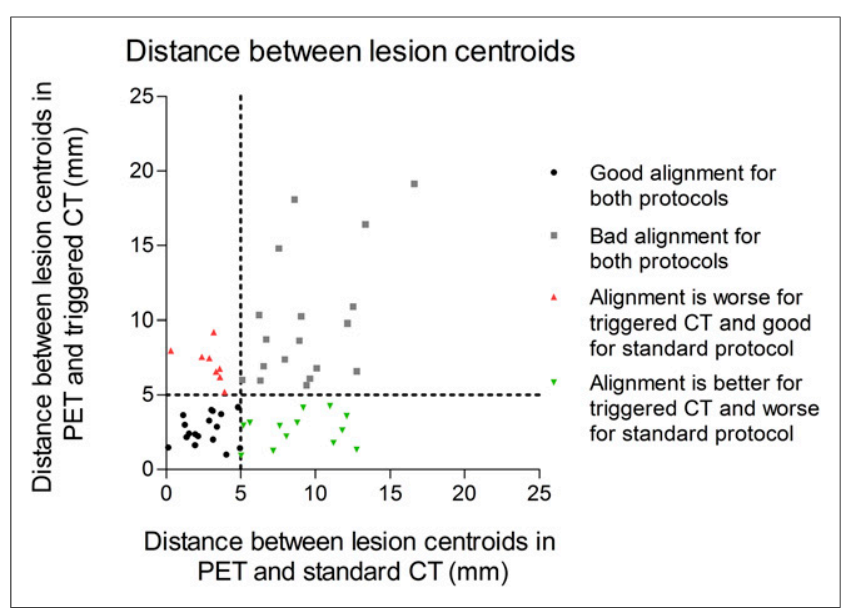

FIGURE 2. Scatterplot showing results of distance between centroids for lesions between triggered CT and standard CT. Lesions with good alignment for both protocols-lesions with a distance between centroids less than $5 \mathrm{~mm}$-are indicated in black. Lesions that showed bad alignment with standard CT protocol $(>5 \mathrm{~mm})$ and improved with triggered CT protocol $(<5 \mathrm{~mm})$ are shown in green. Red indicates lesions where triggered CT became worse (standard CT $<5 \mathrm{~mm}$, triggered $\mathrm{CT}>5 \mathrm{~mm}$ ).

\section{Statistical Analysis}

Because not all the paired groups were normally distributed, statistical analysis was performed with the Wilcoxon signed-rank test using SPSS Statistics 20 (IBM), and statistical significance was defined for a $P$ value less than 0.05 . A Bonferroni adjustment was performed to correct for multiple testing, so all effects are reported at a 0.01 level of significance.

\section{RESULTS}

Thirty-eight patients were included in this study. For 36 patients, the lung-liver boundary was determined and 56 lesions were delineated. The average size of the lesions was $26.4 \pm$ $56.5 \mathrm{~cm}^{3}$ as determined on CT.

The triggered CT scan can result in a considerable improvement of the spatial alignment of the PET and CT images, as shown in Figure 1. The results of the whole study are described in Table 2. There was a significantly better match for the lung-liver boundary when the triggered CT was used in combination with the respirationgated PET than the standard CT and respiration-gated PET, respectively: $4.5 \pm 6.7$ and $9.2 \pm 8.1 \mathrm{~mm}(P<0.001)$. For 22 patients, the difference of the lung-liver boundary for standard CT scans and the PET scan was more than $5 \mathrm{~mm}$. For those patients for whom the mismatch due to motion was greater than $5 \mathrm{~mm}$, the measured prevalence of improved lung-liver boundary matching using the triggered CT was approximately $84 \%$.

The match (Jaccard similarity coefficient and distance between centroids) of the lesions did not show a significant improvement when PET was matched with triggered CT. Not seeing a difference in average values for this comparatively small population, we looked at individual cases. For 6 lesions, there was no overlap of PET and CT (Jaccard similarity coefficient $=0$ ) when the standard CT was used, whereas when the triggered CT was used, these lesions showed overlap. We investigated how often the mismatch between PET and CT was $5 \mathrm{~mm}$ or less (Fig. 2). There was a good match for both standard and triggered CT in 17 lesions. For 22 lesions in the lower and middle lung lobes, a close match (by this definition) was seen for 12 lesions when triggered CT was used, compared with 7 lesions of the standard CT group.

Radiotracer uptake was significantly different when comparing the 2 PET reconstructions: the difference in $\mathrm{SUV}_{\text {max }}$ and $\mathrm{SUV}_{\text {mean }}$ for the PET images reconstructed with the triggered CT compared with the standard CT was $10.9 \pm 6.7$ and $10.5 \pm 6.7(P<0.001)$ for the $\mathrm{SUV}_{\max }$ and $6.4 \pm 4.0$ and $6.1 \pm 4.0(P=0.001)$ for the $\mathrm{SUV}_{\text {mean }}$, respectively. Although there was only a small increment in $\mathrm{SUV}_{\max }$ and $\mathrm{SUV}_{\text {mean }}$ in the PET scans reconstructed with the triggered CT versus the standard CT, for 42 and 41 lesions the $\mathrm{SUV}_{\text {max }}$ and $\mathrm{SUV}_{\text {mean }}$, respectively, were higher for the triggered CT than the standard CT.

The axial range and duration of the triggered CT scan differed between the 2 PET/CT scanners. For the Biograph $40 \mathrm{mCT}$ scanner, the triggered CT had to cover the whole bed position to be able to reconstruct the PET image. This was not the case for the Biograph $64 \mathrm{mCT}$ Flow. As a consequence, the range and the duration of the triggered CT scans were longer for the Biograph $40 \mathrm{mCT}$ than the Biograph 64 mCT Flow: 46.8 (7.0) $\mathrm{cm}$ and 3.1 (1.6) min, respectively, for the Biograph $40 \mathrm{mCT}$ and $32.0(3.9) \mathrm{cm}$ and 1.7 (0.7) min, respectively, for the Biograph $64 \mathrm{mCT}$ Flow.

\section{DISCUSSION}

Our study showed a significantly better alignment of PET and CT when the figure of merit was based on the lung-liver boundary. When we considered lung lesions, in 6 patients there was no overlap at all between the lesions on PET and standard CT, whereas there was overlap on PET and triggered CT in these patients. Unfortunately, we could not demonstrate statistical significance in the lung lesions, probably because of the limited number of patients. Although the SUV increased significantly for the lung lesions, the difference in absolute SUV was small, indicating limited relevance for diagnosis. However, the results indicate that SUV measurements are affected, which can be of importance when measuring therapy response or for the purpose of radiotherapy planning.

The use of continuous-bed-motion acquisition techniques (Biograph $64 \mathrm{mCT}$ Flow) led to shorter setup and acquisition times, which is patient-friendly and will more easily be implemented in

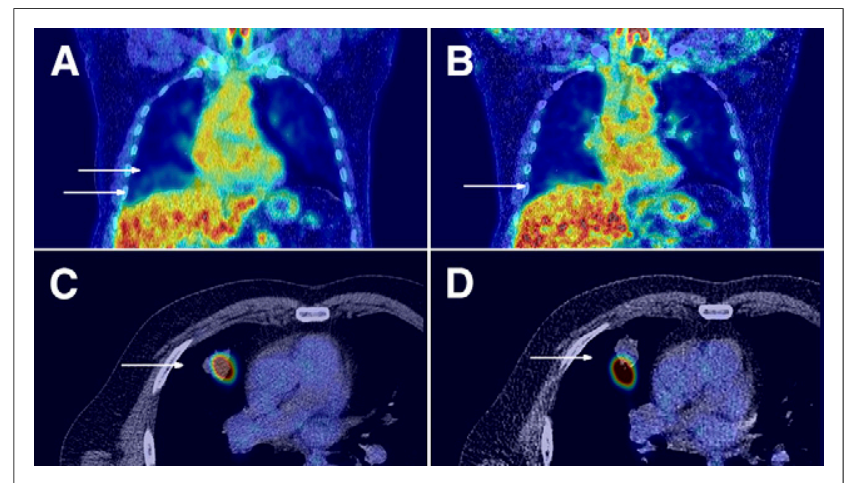

FIGURE 3. Patient with non-small cell lung cancer squamous cell carcinoma in right upper lobe. On left are coronal (A) and axial (C) planes of standard CT and corresponding respiration-gated PET image, on right coronal (B) and axial (D) planes of triggered CT and corresponding PET. Improved match for lung-liver boundary can be seen when triggered $C T$ is used (A and B), but spatial match for PET and CT of lung lesion is worse (C and D). For first PET and standard CT scan (A), 2 arrows were used to indicate lung-liver boundary on PET and CT. Patient was scanned on Biograph 40 mCT scanner. 


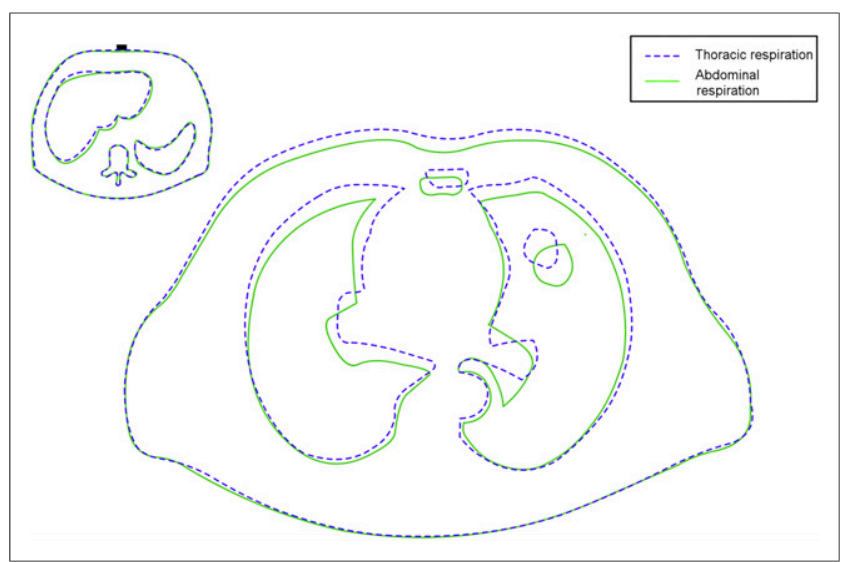

FIGURE 4. Illustration of mismatch in 2 scans caused by difference in breathing. One scan was made during thoracic respiration (dashed line) and 1 during abdominal respiration (solid line). Pressure sensor is located at same position, indicating that the 2 scans were acquired at similar time during respiratory cycle (left corner). Excursion of abdominal wall at location of pressure sensor differs from that at position at thoracic wall, suggesting less effective tracking of respiratory motion. Even though scans were made during same respiratory phase, it did not result in better match for lung lesion.

routine clinical care. These time savings were a direct result of the selection of axial range in which gating would be applied. For standard step-and-shoot imaging, extending the axial selection past a single bed position requires the addition of a full bed. This is not the case for continuous-bed-motion acquisition. The average acquisition time of the triggered CT was $3 \mathrm{~min}$ and $7 \mathrm{~s}$ for the Biograph $40 \mathrm{mCT}$ scanner and $1 \mathrm{~min}$ and $39 \mathrm{~s}$ for the Biograph 64 mCT Flow scanner (Table 1).

Although the protocol for triggered CT was comparatively easy for a skilled technologist or physicist, it was not integrated in the workstation and required 1-2 min extra time during the examination. In our prototype, we used an additional computer to determine the trigger points and manually match the respiratory signal settings to the amplitude range determined by the PET workstation. The additional computer was the same one that has long been used in AZ-733V-based PET and CT, for example, gated CT. The manual adjustment caused some uncertainty in the measurement of the trigger points. We do not know if the uncertainty would have clinical importance.

In some cases, when PET and standard CT were compared with PET and triggered CT, the matching was improved in the liver but degraded in the lung. This phenomenon was observed in 5 patients in the group scanned with the Biograph $40 \mathrm{mCT}$ and in 1 patient scanned with the Biograph $64 \mathrm{mCT}$ Flow scanner (Fig. 3). This might be caused by the respiratory tracking method, using a pressure sensor in a belt located around the patient's abdomen (22). Movement of the different areas in the thorax, however, is more complex and cannot be accurately measured with 1 abdominal belt sensor (Fig. 4). Other tracking methods might be more appropriate for lesions in different regions in the lungs, such as methods that track the respiration of the patient by measuring the breathed airflow (spirometer) or changing temperature of breathed air (temperature sensor) $(10,23)$. However, these methods are not commonly used in clinical routine (23). Another method to gate the PET data is to use reconstructed images with a high time resolution and to use a (elastic) transformation to match each image, known as list-mode gating $(24,25)$. However, this method is not yet implemented in the clinical routine and still has some problems to overcome, for instance, the noise level in the data (23). This suggests that more research is needed to find the best respiratory tracking method for clinical use.

Should the combination of respiration-gated PET and triggered CT be used clinically, considering that it has the potential to match the modalities precisely? It is reasonable and important to improve the match, if it can be done conveniently and without unwarranted additional time or radiation dose to the patient. Our protocol includes a standard CT, followed by PET, followed by a prospectively triggered CT scan. There is room for improvement in 3 areas. First, to keep the x-ray dose as low as reasonably achievable, the standard scan can be skipped in areas that are also scanned in triggered CT. Second, the scan duration for triggered CT is long. Triggered CT can be replaced with a spiral acquisition during a breath-hold that has been amplitude-matched to the PET scan. We are examining this possibility and hope to report our results in the near future. Third, this research protocol can be integrated into the scanner's hardware and software.

The possibility of performing CT after PET, instead of before PET, is a novel aspect of our protocol. Because optimal gating of PET depends on the patient's breathing patterns during the PET scan, a CT scan that matches the optimally gated PET can be realized in just 1 of 4 ways: using free-breathing CT (the usual approach) or CT under appropriate breathing instructions; by acquiring CT data during all phases of breathing, followed by matching retrospectively; with slow or time-averaged CT; or with the described prospective approach. The first approach, however, does not guarantee to provide a proper match. The second and third methods typically require a high x-ray dose. Our novel prospective method has the potential to provide both a good match and an LD setting suitable for routine clinical use.

\section{CONCLUSION}

Respiration-optimal-gated PET in combination with a respirationtriggered CT scan resulted in a significantly better match in the region of the liver dome and a significantly higher SUV for lesions in the lungs. In every lung tumor considered, the new technique based on triggered CT provided partial overlap of PET and CT, whereas the standard approach based on spiral CT sometimes failed. However, we were unable to demonstrate a statistically significant improvement in the alignment of PET and CT for lung lesions. A different respiratory tracking method might be the key to making the method robust.

\section{DISCLOSURE}

The costs of publication of this article were defrayed in part by the payment of page charges. Therefore, and solely to indicate this fact, this article is hereby marked "advertisement" in accordance with 18 USC section 1734. Willem Grootjans received an educational grant during the writing of this manuscript from Siemens Healthcare, the Hague, The Netherlands. The University of Tennessee has an active collaboration agreement with Siemens Healthcare. Dustin R. Osborne and Shelley Acuff occasionally provide expert testimonial for Siemens Healthcare. James J. Hamill is employed by Siemens Healthcare. No other potential conflict of interest relevant to this article was reported. 


\section{ACKNOWLEDGMENT}

We thank Matt Mitchell (Siemens) for the engineering implementation.

\section{REFERENCES}

1. Bomanji JB, Costa DC, Ell PJ. Clinical role of positron emission tomography in oncology. Lancet Oncol. 2001;2:157-164.

2. Fletcher JW, Djulbegovic B, Soares HP, et al. Recommendations on the use of ${ }^{18}$ F-FDG PET in oncology. J Nucl Med. 2008;49:480-508.

3. Bettinardi V, Picchio M, Di Muzio N, Gilardi MC. Motion management in positron emission tomography/computed tomography for radiation treatment planning. Semin Nucl Med. 2012;42:289-307.

4. Berghmans T, Dusart M, Paesmans M, et al. Primary tumor standardized uptake value (SUVmax) measured on fluorodeoxyglucose positron emission tomography (FDG-PET) is of prognostic value for survival in non-small cell lung cancer (NSCLC): a systematic review and meta-analysis (MA) by the European Lung Cancer Working Party for the IASLC Lung Cancer Staging Project. $J$ Thorac Oncol. 2008;3:6-12.

5. Zaizen Y, Azuma K, Kurata S, et al. Prognostic significance of total lesion glycolysis in patients with advanced non-small cell lung cancer receiving chemotherapy. Eur J Radiol. 2012;81:4179-4184.

6. Chen HH, Chiu NT, Su WC, Guo HR, Lee BF. Prognostic value of whole-body total lesion glycolysis at pretreatment FDG PET/CT in non-small cell lung cancer. Radiology. 2012;264:559-566.

7. Im HJ, Pak K, Cheon GJ, et al. Prognostic value of volumetric parameters of ${ }^{18} \mathrm{~F}$ FDG PET in non-small-cell lung cancer: a meta-analysis. Eur J Nucl Med Mol Imaging. 2015;42:241-251.

8. Callahan J, Binns D, Dunn L, Kron T. Motion effects on SUV and lesion volume in 3D and 4D PET scanning. Australas Phys Eng Sci Med. 2011;34:489-495.

9. Daouk J, Fin L, Bailly P, Meyer ME. Improved attenuation correction via appropriate selection of respiratory-correlated PET data. Comput Methods Programs Biomed. 2008;92:90-98.

10. Nehmeh SA, Erdi YE. Respiratory motion in positron emission tomography/ computed tomography: a review. Semin Nucl Med. 2008;38:167-176.

11. Grootjans W, de Geus-Oei LF, Meeuwis AP, et al. Amplitude-based optimal respiratory gating in positron emission tomography in patients with primary lung cancer. Eur Radiol. 2014;24:3242-3250.
12. van der Vos CS, Grootjans W, Meeuwis AP, et al. Comparison of a free-breathing CT and an expiratory breath-hold CT with regard to spatial alignment of amplitude-based respiratory-gated PET and CT images. J Nucl Med Technol. 2014;42:269-273.

13. Fin L, Daouk J, Morvan J, et al. Initial clinical results for breath-hold CT-based processing of respiratory-gated PET acquisitions. Eur J Nucl Med Mol Imaging. 2008;35:1971-1980.

14. Osman MM, Cohade C, Nakamoto Y, Wahl RL. Respiratory motion artifacts on PET emission images obtained using CT attenuation correction on PET-CT. Eur J Nucl Med Mol Imaging. 2003;30:603-606.

15. Nehmeh SA, Erdi YE, Meirelles GS, et al. Deep-inspiration breath-hold PET/CT of the thorax. J Nucl Med. 2007;48:22-26.

16. Grgic A, Ballek E, Fleckenstein J, et al. Impact of rigid and nonrigid registration on the determination of ${ }^{18} \mathrm{~F}$-FDG PET-based tumour volume and standardized uptake value in patients with lung cancer. Eur J Nucl Med Mol Imaging. 2011;38:856-864.

17. van Elmpt W, Hamill J, Jones J, De Ruysscher D, Lambin P, Ollers M. Optimal gating compared to 3D and 4D PET reconstruction for characterization of lung tumours. Eur J Nucl Med Mol Imaging. 2011;38:843-855.

18. FDG-PET/CT Accreditation. resEARch 4 Life: an EANM initiative website. http://earl.eanm.org/cms/website.php?id=/en/projects/fdg_pet_ct_accreditation. htm. Accessed September 13, 2015.

19. Panin VY, Smith AM, Hu J, Kehren F, Casey ME. Continuous bed motion on clinical scanner: design, data correction, and reconstruction. Phys Med Biol. 2014;59:6153-6174.

20. Li XA, Stepaniak C, Gore E. Technical and dosimetric aspects of respiratory gating using a pressure-sensor motion monitoring system. Med Phys. 2006;33:145-154.

21. Erdi YE, Mawlawi O, Larson SM, et al. Segmentation of lung lesion volume by adaptive positron emission tomography image thresholding. Cancer. 1997;80 (12, suppl):2505-2509.

22. Dasari PK, Shazeeb MS, Konik A, et al. Adaptation of the modified Bouc-Wen model to compensate for hysteresis in respiratory motion for the list-mode binning of cardiac SPECT and PET acquisitions: testing using MRI. Med Phys. 2014;41:112508.

23. Pépin A, Daouk J, Bailly P, Hapdey S, Meyer ME. Management of respiratory motion in PET/computed tomography: the state of the art. Nucl Med Commun. 2014;35:113-122.

24. Lamare F, Ledesma Carbayo MJ, Cresson T, et al. List-mode-based reconstruction for respiratory motion correction in PET using non-rigid body transformations. Phys Med Biol. 2007;52:5187-5204.

25. Sun T, Mok GS. Techniques for respiration-induced artifacts reductions in thoracic PET/CT. Quant Imaging Med Surg. 2012;2:46-52. 